\title{
BeppoSAX observations of PKS 0528+134
}

G. Ghisellini, G. Tagliaferri, L. Costamante, L. Maraschi; ${ }^{a}$ A. Celotti, G. Fossati; ${ }^{\text {b }}$ L. Bassani, M. Cappi F. Frontera, E. Pian; ${ }^{c}$ A. Comastri; ${ }^{d}$ M. Cavallone, G. De Francesco, L. Lanteri, C.M. Raiteri, G. Sobrito, M. Villata; ${ }^{\text {e }}$ S. Giarrusso, B. Sacco; ${ }^{\mathrm{f}}$ I.S. Glass; ${ }^{\mathrm{g}}$ P. Grandi; ${ }^{\text {h }}$ E. Massaro; ${ }^{\mathrm{i}}$ S. Molendi; ${ }^{j}$ P. Padovani; ${ }^{k}$ G.G.C. Palumbo; ${ }^{1}$ C. Perola; ${ }^{m}$ M. Salvati; ${ }^{n}$ A. Treves; ${ }^{\circ}$

${ }^{a}$ Brera Astronomical Observatory, V. Bianchi, 46 Merate, Italy

${ }^{\mathrm{b}}$ SISSA, Trieste, Italy

${ }^{\mathrm{c}}$ TESRE Bologna, Italy

${ }^{\mathrm{d} O s s e r v . ~ A s t r o n . ~ o f ~ B o l o g n a, ~ I t a l y ~}$

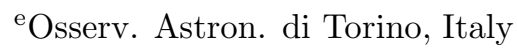

${ }^{\mathrm{f}} \mathrm{IFCAI} / \mathrm{CNR}$, Palermo, Italy

${ }^{\mathrm{g}}$ South African Astron. Observ.

${ }^{\mathrm{h}} \mathrm{ISA} / \mathrm{CNR}$, Frascati, Roma, Italy

${ }^{\mathrm{i}}$ Univ. of Rome La Sapienza, Italy

jIFCTR-CNR Milano, Italy

${ }^{\mathrm{k} E S A-S T S c I, ~ B a l t i m o r e, ~ U S A ~}$

${ }^{1}$ Univ. of Bologna, Italy

${ }^{\mathrm{m}}$ Univ. of Rome III, Italy

${ }^{\mathrm{n}}$ Arcetri Astroph. Obs., Italy

${ }^{\circ}$ Univ. of Como, Italy

We report on the BeppoSAX observations of the $\gamma$-ray blazar PKS 0528+134 performed in Feb and Mar 1997, during a multiwavelength campaign involving EGRET and ground based telescopes. The source was in a faint and hard state, with energy spectral index $\alpha=0.48 \pm 0.04$ between 0.1 and $10 \mathrm{keV}$, and [2-10] keV flux of $2.7 \times 10^{-12} \mathrm{erg} \mathrm{cm}^{-2} \mathrm{~s}^{-1}$. No significant variability was observed. The source was detected in the $20-120 \mathrm{keV}$ band by the PDS, with a flux lying slightly above the extrapolation from lower $\mathrm{X}$-ray energies. Comparing this low state with previous higher states of the source, there is an indication that the $\mathrm{X}$-ray spectrum hardens and the $\gamma$-ray spectrum steepens when the source is fainter.

\section{PKS $0528+134$}

PKS $0528+134(z=2.07)$ is the second most distant quasar detected by EGRET in the $\gamma$-ray band. Located in the galactic anticenter region, it is heavily absorbed, although the estimates of $A_{V}$ are very uncertain (ranging from 2.3 to 5 ). Thus PKS $0528+134$ is faint in the optical, with a typical average magnitude of 19.5 in the $V$ band. It is a strong and flat radio source, with detected superluminal motion (with $\beta_{a p p} \sim 4$, [2]). Close to Geminga and the Crab, it was frequently ob- 


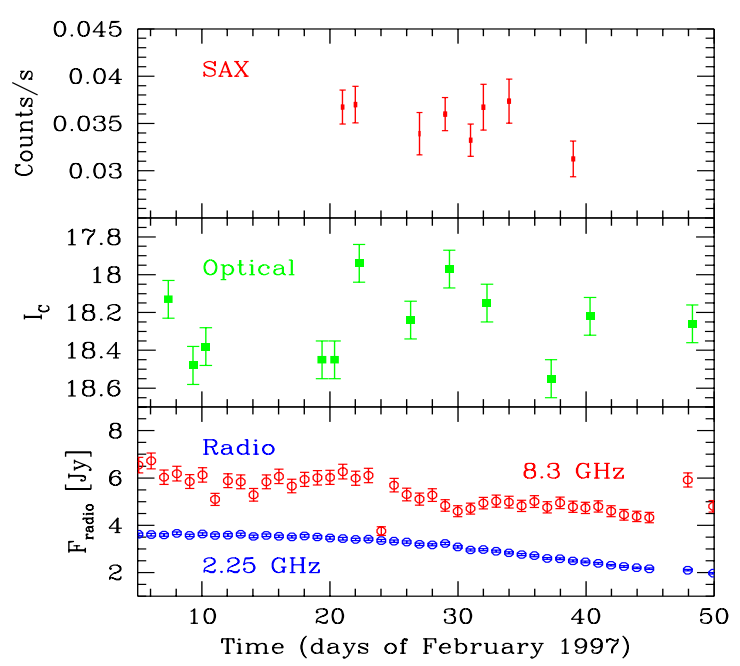

Figure 1. Top panel: BeppoSAX MECS light curve of PKS $0528+134$ corresponding to the 8 observations. Middle panel: the optical light curve in the same period, in the $I_{C}$ filter. Bottom panel: the light curve at 8.3 and $2.25 \mathrm{GHz}$, by the Green Bank monitoring campaign.

served by EGRET and seen to flare in March 1993, when the $\gamma$-ray flux was 3 times brighter than average. Flux changes in the $\gamma$-band are accompanied by spectral variations in the sense that the spectrum is harder when brighter [3].

\section{RESULTS}

BeppoSAX observed the source 8 times between Feb 21 and Mar 11, 1997, within a multiwavelength observing campaign involving also EGRET and ground based telescopes.

Light curves - In Fig. 1 we show the MECS light curve corresponding to the 8 SAX observations. The flux is constant (probability of $25 \%$ to obtain a larger value of $\chi^{2}$, when fitting a constant). No significant variations are present within the single observations. On the contrary, small amplitude variations are detected in the optical and at $8.3 \mathrm{GHz}$, as shown in the middle and lower panel of Fig. 1.

Spectral fitting - Since the flux is constant we decided to add together the 8 spectra. A single power law fit to the LECS+MECS data (see Fig. 2 ) yields an energy spectral index $\alpha=0.44 \pm 0.06$

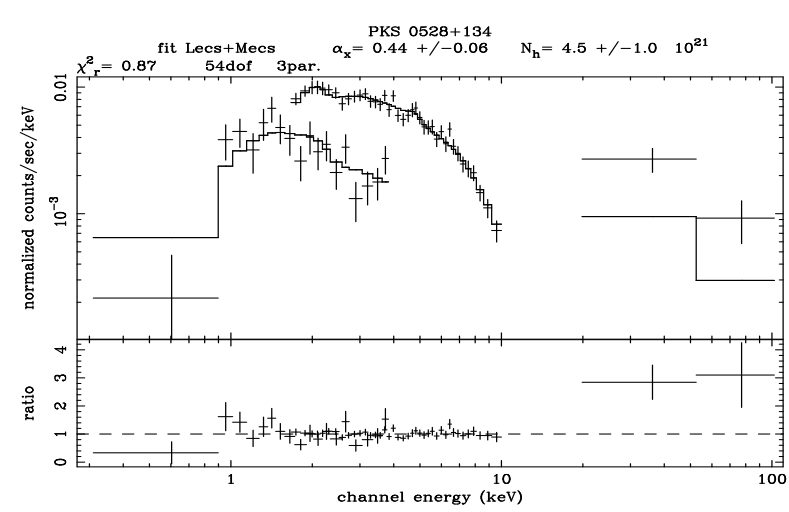

Figure 2. Fit to the LECS and MECS BeppoSAX spectrum of PKS $0528+134$. PDS data are not used for the fit, and appear to lie above the extrapolation of the power law from lower energies.

and $N_{H}=(4.5 \pm 1.0) \times 10^{21} \mathrm{~cm}^{-2}$, with $\chi_{r}^{2}=0.87$ and 54 degree of freedom. Note that, as shown in Fig. 2, the two PDS data points lye above the model fit.

A long standing problem with this source is the determination of its optical extinction and the column $N_{H}$ along the line of sight. The value that we found is in good agreement with the sum of the estimated absorption caused by the column of neutral galactic hydrogen $\left[N_{H}=(2.6 \pm 0.1) \times 10^{21}\right.$ $\left.\mathrm{cm}^{-2}\right]$, plus that due to the outer edge of the molecular cloud Barnard 30 in the $\lambda$ Orion rings of clouds $\left[N_{H} \sim 1.3 \times 10^{21} \mathrm{~cm}^{-2}\right]$. The best fit value found by published ASCA observations, $N_{H} \sim 5 \times 10^{21} \mathrm{~cm}^{-2}[3]$, is in good agreement with our value. In addition to the ASCA published spectra, we have re-analyzed two other ASCA observations performed in 1995, taken from the ASCA archive. Fitting the sum of 3 ASCA observations performed in a time interval of 2 weeks, when the source was bright, we found a value of $N_{H}=(5.3 \pm 0.1) \times 10^{21} \mathrm{~cm}^{-2}$, which is the best measure of the column so far.

Since the BeppoSAX measurement is consistent with this value, we decided, when investigating the flux-slope correlation, to fix the $N_{H}$ at this value. In this case the LECS+MECS data are fitted by a single power law with an energy index $\alpha=0.48 \pm 0.04$.

Including the PDS data and using a single 


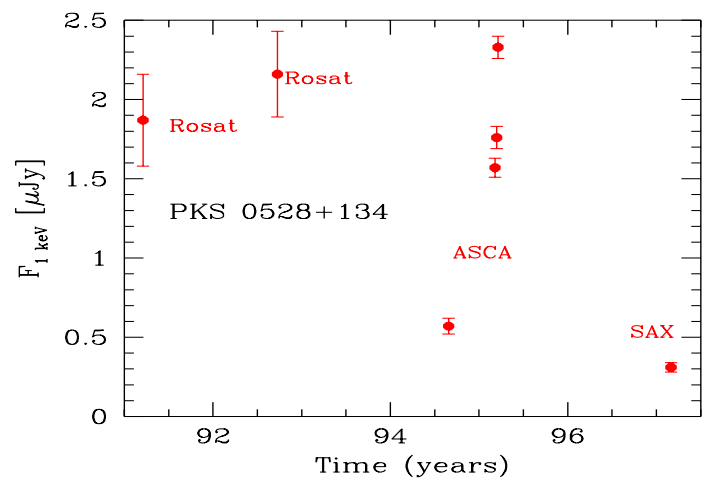

Figure 3. Historical light curve in the X-ray band, after 1991. All data have been reanalyzed assuming a fixed $N_{H}=5.3 \times 10^{21} \mathrm{~cm}^{-2}$.

power law fit we obtain $\alpha=0.44 \pm 0.04$, with the PDS points still lying somewhat above the model fit. The same data can be fitted with a broken power law model with $\alpha_{1}=0.46 \pm 0.04$ and $\alpha_{2}=-0.15 \pm 0.29$ and break energy $E_{B} \sim 10_{-3}^{+12}$ $\mathrm{keV}$. The latter model significantly improves the fit, according to the $F$-test (99\%).

Historical $\mathbf{X}$-ray light curve - In Fig. 3 we show the light curves of all the observations in the X-ray band after 1991. In 1995 the source varied by $50 \%$ in 2 weeks; while between the 1995 ASCA and our 1997 BeppoSAX observations, when the source was at its faintest historical level, the flux decreased by a factor $\sim 7$. In the $\gamma-$ ray band the source was observed more often, with a variability of a factor 13 in 2 months, and a factor $\sim 2$ in 2 days during the flare occured in 1993 (see [1]).

Flux-spectral index correlations - In Fig. 4 (upper panel) we report the $\mathrm{X}$-ray spectral index determined by BeppoSAX and ASCA vs the $1 \mathrm{keV}$ flux. There is an indication of a flattening of the slope when the source is fainter, just the opposite of what happens in the $\gamma$-ray band (lower panel). While in the $\gamma$-ray band this behaviour is similar to what observed in other sources (see e.g. [4]), the 'flatter when fainter' behaviour in the $\mathrm{X}^{-}$ ray band is unusual, and likely to yield important informations and/or constraints on the emission models.

\section{THE SED}

In Fig. 5 we show the overall spectral distribution of PKS $0528+134$ corrisponding to differ-
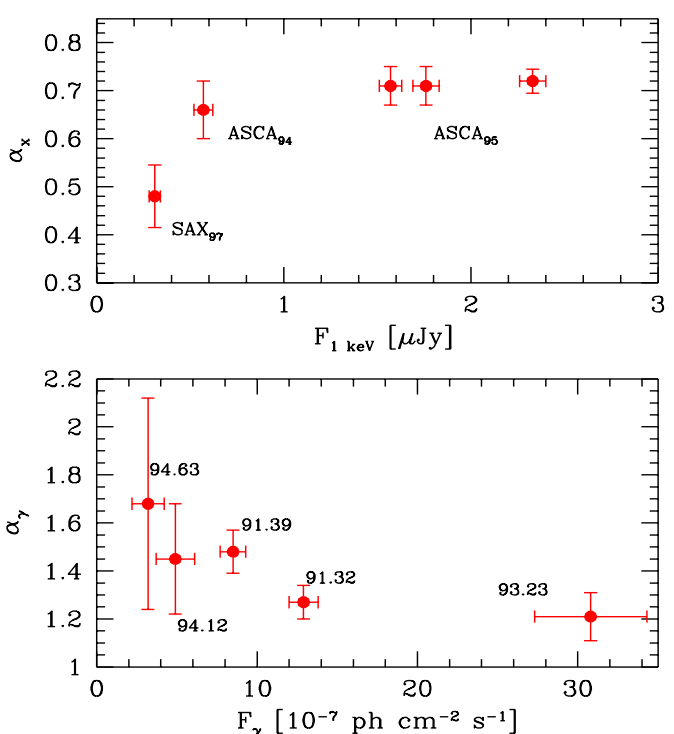

Figure 4. Spectral indices vs flux in the $\mathrm{X}$-ray and $\gamma$-ray bands. All X-ray data have been reanalyzed assuming a fixed $N_{H}=5.3 \times 10^{21} \mathrm{~cm}^{-2}$.

ent epochs, as indicated by the labels. Data have been dereddened assuming $N_{H}=5.3 \times 10^{21} \mathrm{~cm}^{-2}$, corresponding to $A_{V}=3$. As all other $\gamma$-ray bright blazars, also the SED of PKS 0528+134 is characterized by two peaks, one in the far IR region, and the other one at $\sim 10 \mathrm{MeV}$. As discussed in [3], the dereddened optical spectrum is inverted $\left(\alpha_{o}=-0.18 \pm 0.08\right)$, and thus indicative of the presence of the blue-bump. Note that the $\mathrm{MeV}-\mathrm{GeV}$ emission dominates the bolometric output by a large amount, reaching a luminosity in excess of $10^{49} \mathrm{erg} \mathrm{s}^{-1}$.

\section{DISCUSSION}

Among the many proposed models for $\gamma-$ ray bright blazars, the most popular are the synchrotron-self Compton (SSC) and the external Compton (EC) models. In both of them the synchrotron radiation is responsible for the low energy emission (up to the optical-UV band), while the inverse Compton process forms the $\mathrm{X}$ to the $\gamma$-ray spectrum. What differs in the two models is the nature of the seed photons to be Comptonized at high energies. They are locally produced synchrotron photons in the SSC model, while in the EC model the main contribution to 
4

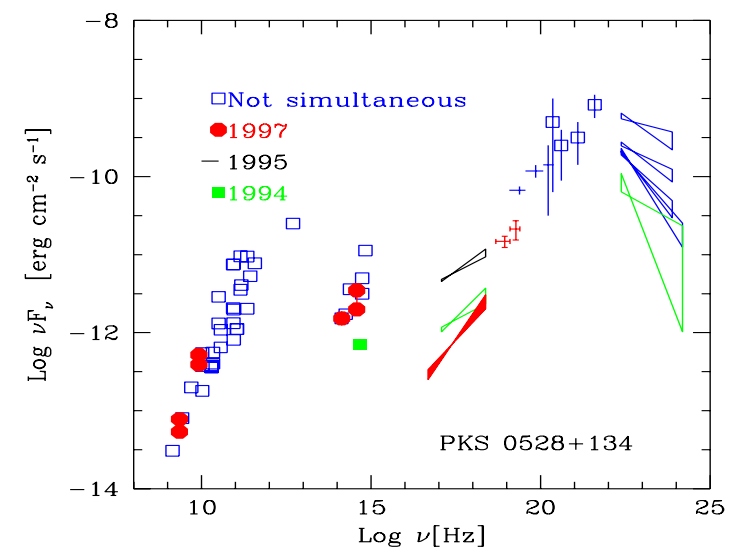

Figure 5. Overall spectral energy distribution of PKS 0528+134. Simultaneous observations are marked by the different symbols, as labelled. Other non-simultaneous data are taken from the literature.

the seed photons comes from regions external to the $\gamma$-ray producing zone. The presence of the blue-bump and of luminous emission lines supports, in the case of PKS $0528+134$, the EC model. For a detailed discussion of the fitting of the 1994 overall spectrum with SSC and EC models see [3]. If confirmed, the variability behaviour in the high energy band (X-ray spectrum steeper when brighter; $\gamma$-ray spectrum steeper when fainter) could help to understand the role of the SSC vs the EC model, and possibly to shed light to the importance of electron-positron pair production. We can envisage two scenarios to explain the observed behaviour:

1) The $X$-rays are produced by both the self Compton and EC processes. There are two typical frequencies of the seed photons: one corresponding to the peak of the synchrotron emission (in the far IR), and one corresponding to the external radiation. If emission lines and blue bump photons form the bulk of the external radiation, their typical frequency, as observed in the comoving frame, is in the far UV. If the radiation energy densities of these two components are comparable (within a factor 10), then the self Compton spectrum will dominate at lower $\mathrm{X}$-ray energies, while the EC spectrum dominates above, being entirely responsible for the emission at $\gamma-$ ray energies. This is because the self Compton spectrum is somewhat steeper than the EC one produced by the same electrons, and because the maximum self Compton frequency is lower. If the number of emitting electrons increases, then the synchrotron and the EC flux vary linearly, while the self Compton flux varies quadratically, making it to dominate the flux over a larger $\mathrm{X}$ ray energy range. This could explain the 'steeper when brighter' behaviour of PKS $0528+134$ in the $\mathrm{X}$-rays. To explain the opposite behaviour in the $\gamma$-rays it is necessary to assume, in addition, that the variation of the electron number is accompanied by a flattening of the high energy tail of their energy distribution, a behaviour often invoked to explain blazar variability.

2) Another process capable to account for the $\mathrm{X}$-ray 'steeper when brighter' behaviour is $\mathrm{e}^{ \pm}$ pair production. Suppose that, when the source is in a high (and hard) $\gamma$-ray state, a small fraction of high energy power gets converted in electron positron pairs, making the particle energy distribution to steepen toward lower energies. This could account for a brighter and steeper medium energy X-ray spectrum, while at higher energies the emission of the 'normal' particles (with a flatter energy distribution) dominates. The main difficulty of this scenario is a 'fine tuning' problem, since the high energy power that is absorbed and reprocessed must be of the right amount (some per cent at most). If it is less, then pairs are not produced in a sufficient amount to contribute anywhere in the spectrum. If it is higher, then they could increase the amount of target photons for $\gamma-\gamma$ collisions, inducing a catastrofic cascade, and an overproduction of $\mathrm{X}$-ray with respect to what we observe. A detailed investigation of these ideas is in progress and will be presented elsewhere.

\section{REFERENCES}

1. Mukherjee, R. et al. 1996, ApJ, 470, 831

2. Pohl, M., Reich W., Schlickeiser R., Reich P. \& Ungerechts H., 1996, A\&AS 120, 529

3. Sambruna, R.M. et al., 1997, ApJ, 474, 639 